\title{
Correction: Bougma, K., et al. Iodine and Mental Development of Children 5 Years Old and Under: A Systematic Review and Meta-Analysis. Nutrients 2013, 5, 1384-1416
}

\author{
Karim Bougma ${ }^{1}$, Frances E. Aboud ${ }^{2}$, Kimberly B. Harding ${ }^{3}$ and Grace S. Marquis ${ }^{1, *}$ \\ 1 School of Dietetics and Human Nutrition, McGill University, 21111 Lakeshore Road, CINE \\ Building, Sainte Anne-de-Bellevue, QC, H9X 3V9, Canada; \\ E-Mail: karim.bougma@mail.mcgill.ca \\ 2 Department of Psychology, McGill University, 1205 Dr. Penfield Avenue, Montreal, QC, \\ H3A 1B1, Canada; E-Mail: frances.aboud@mcgill.ca \\ 3 Micronutrient Initiative, 180 Elgin Street, Suite 1000, Ottawa, ON, K2P 2K3, Canada; \\ E-Mail: kharding@micronutrient.org
}

* Author to whom correspondence should be addressed; E-Mail: grace.marquis@mcgill.ca;

Tel.: +1-514-398-7839; Fax: +1-514-398-1020.

Received: 24 November 2014 / Accepted: 25 November 2014 / Published: 10 December 2014

We have found an inadvertent error in our paper published in Nutrients [1]. One standard error was not converted into standard deviation and this affected the following effect sizes and intelligence quotient (IQ):

On page 1384, the sentences “Average effect sizes for these four designs were $\mathbf{0 . 6 8}$ (2 RCT studies), 0.46 (8 non-RCT studies), 0.52 (9 cohort stratified by mothers’ iodine status), and 0.54 (4 cohort stratified by infants' iodine status). This translates into 6.9 to 10.2 IQ points lower in iodine deficient children compared with iodine replete children" should be "Average effect sizes for these four designs were 0.48 (2 RCT studies), 0.46 (8 non-RCT studies), 0.52 (9 cohort stratified by mothers' iodine status), and 0.54 (4 cohort stratified by infants' iodine status). This translates into 6.9 to 8.1 IQ points lower in iodine deficient children compared with iodine replete children”.

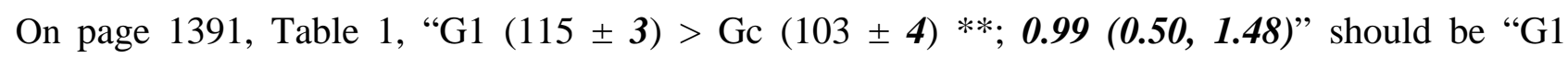
$(115 \pm$ 18.7 $)>$ Gc $(103 \pm 24.0) * * ; 0.56(0.09,1.02) ”$.

On page 1400, the sentences "Thilly et al. [52-55] found that supplementation during the second and third trimester with a single dose of $475 \mathrm{mg}$ of iodine resulted in a statistically significant difference in children's mental outcome compared to the placebo control group (Mean 115 vs. 103; $p<0.01$, $\mathrm{d}=\mathbf{0 . 9 9}$ ). The average effect size from these two double blind RCT was $d=\mathbf{0 . 6 8}$." should be 
"Thilly et al. [52-55] found that supplementation during the second and third trimester with a single dose of $\mathbf{4 7 5} \mathrm{mg}$ of iodine resulted in a statistically significant difference in children's mental outcome compared to the placebo control group (Mean 115 vs. 103; $p<0.01, \mathrm{~d}=\mathbf{0 . 5 6}$ ). The average effect size from these two double blind RCT was $d=\mathbf{0 . 4 8}$ ".

On page 1401, the sentence "The average effect size for randomized $(\mathrm{d}=\mathbf{0 . 6 8})$ and non-randomized ( $d=0.46)$ designs was $\mathbf{0 . 4 9}$." should be "The average effect size for randomized $(\mathrm{d}=\mathbf{0 . 4 8}$ ) and non-randomized $(\mathrm{d}=0.46)$ designs was 0.50 .”

On page 1402, the sentence "Consequently, an effect size of $\mathbf{0 . 4 9}$ translates into a 7.4 IQ point difference $(\mathbf{0 . 4 9} \times 15)$ between groups." should be "Consequently, an effect size of $\mathbf{0 . 5 0}$ translates into a 7.5 IQ point difference $(0.50 \times 15)$ between groups.”

On page 1402, Figure 2 title, “ $(\mathrm{Q}=\mathbf{5 4 . 8 1}$, $\mathrm{df}=15, p<0.0001)$ ” should be “( $\mathrm{Q}=\mathbf{5 4 . 5 7}$, $\mathrm{df}=15$, $p<0.0001)$ ".

On page 1405, the sentence "The average effect size for the two randomized controlled studies was $\mathrm{d}=\mathbf{0 . 6 8}$ and for the eight non-randomized studies was $\mathrm{d}=0.46$ " should be "The average effect size for the two randomized controlled studies was $\mathrm{d}=\mathbf{0 . 4 8}$ and for the eight non-randomized studies was $\mathrm{d}=0.46 ”$.

On page 1406, the sentence "The mean effect size for supplementation studies was therefore $\mathbf{0 . 4 9}$ which translates into 7.4 IQ points assuming a SD of 15" should be "The mean effect size for supplementation studies was therefore $\mathbf{0 . 5 0}$ which translates into 7.5 IQ points assuming a SD of 15”.

On page 1409, the sentence "We believe that the best estimate to date of the effect size of iodine supplementation on mental development in children 5 years old and under is $\mathbf{0 . 4 9}$, which translates into 7.4 IQ points lost due to iodine deficiency." Should be "We believe that the best estimate to date of the effect size of iodine supplementation on mental development in children 5 years old and under is $\mathbf{0 . 5 0}$, which translates into 7.5 IQ points lost due to iodine deficiency.”

These changes have no material impact on the conclusions of our paper. We apologize to our readers.

\section{Reference}

1. Bougma, K.; Aboud, F.E.; Harding, K.B.; Marquis, G.S. Iodine and Mental Development of Children 5 Years Old and Under: A Systematic Review and Meta-Analysis. Nutrients 2013, 5, 1384-1416.

(C) 2014 by the authors; licensee MDPI, Basel, Switzerland. This article is an open access article distributed under the terms and conditions of the Creative Commons Attribution license (http://creativecommons.org/licenses/by/4.0/). 